\title{
Competencias psicosociales en estudiantes universitarios
}

\author{
PSYCHOSOCIAL COMPETENCES IN UNIVERSITY STUDENTS
}

\author{
Deleana Paola Rincón Fuenmayor
}

deleana@gmail.com

Código ORCID: 0000-0001-8438-0044

Universidad del Zulia, Venezuela

Recibido abril 2019 | Arbitrado mayo 2019 | Publicado julio 2019

Resumen

Palabras clave:

Orientación; competencias; psicosociales; estudiantes universitarios
La investigación tiene como objetivo analizar las competencias psicosociales de los estudiantes del Instituto Universitario de Tecnología READIC UNIR, basándose en los planteamientos de Chickering y Reisser (1993). El tipo de investigación es descriptiva no experimental, de campo, transeccional, con una población de 25 estudiantes universitarios. Se diseñó un instrumento tipo Likert, con una confiabilidad de 0,89 . Los resultados reflejan que el $92 \%$ de los estudiantes, poseen en un rango alto las competencias psicosociales, el $8 \%$ medianamente poseen esta dimensión. La media de la dimensión es de 4.36 considerándose su competencia en un nivel "Alto", revelando que los estudiantes poseen un grado de suficiencia y responsabilidad para avanzar por la universidad y realizar tareas psicosociales. Se muestran en muchas formas con competencias psicosociales que otorgan conciencia, estabilidad, destreza, confianza e integración, ya que cada vector comprende fortalezas y debilidades para el crecimiento y para adaptarse cuando aparecen los problemas.

\section{Abstract}

\section{Keywords:}

Orientation; competences; psychosocial; university students
The objective of the research is to analyze the psychosocial competences of the students of the READIC UNIR University Institute of Technology, based on the Chickering and Reisser (1993) approaches. The type of research is descriptive, non-experimental, field, transectional, with a population of 25 university students. A Likert-type instrument was designed, with a reliability of 0.89 . The results reflect that $92 \%$ of the students possess psychosocial competences in a high range, $8 \%$ moderately possess this dimension. The mean of the dimension is 4.36, considering their competence at a "High" level, revealing that students possess a degree of proficiency and responsibility to advance through the university and perform psychosocial tasks. They are shown in many ways with psychosocial competences that provide awareness, stability, skill, confidence and integration, since each vector includes strengths and weaknesses for growth and for adapting when problems arise. 


\section{INTRODUCCIÓN}

El presente artículo comprende elementos constitutivos al estudio de las competencias psicosociales que desarrollan los estudiantes universitarios en el Instituto Tecnológico Readic UNIR lo que hace una relación clara con este estudio ya que comprende la descripción de los aspectos psicosociales como fuente de preparación para el campo laboral, sabiendo que en el mismo se requiere de profesionales flexibles, autónomos y emprendedores para contribuir al crecimiento económico y a la diversidad cultural.

Es pertinente acotar que la Unesco (2009) considera que la educación es un derecho humano para todos, a lo largo de toda la vida, y que el acceso a la instrucción debe ir acompañado de la calidad. La Unesco es la única organización de las Naciones Unidas que dispone de un mandato para abarcar todos los aspectos de la educación.

La educación superior supone uno de los ámbitos más propicios para desarrollar personas que generen un alto impacto en la comunidad, para educar integralmente a estudiantes, por lo tanto las universidades deben adquirir una filosofía de desarrollo desde la cual se vea y se valore al estudiante como una totalidad, no se debe pensar que los estudiantes únicamente buscan pasar los exámenes y graduarse, en realidad ellos adquieren conocimientos, desarrollan actitudes, valores, consolidan competencias para la vida laboral y se aspira que logren ser mejores ciudadanos y personas.

Se deben contratar docentes que sepan cómo lograr ese desarrollo para que estas instituciones no se conviertan únicamente en un dispensario de servicios. Se requieren universidades que transfieran conocimientos relevantes y generen responsabilidad social, creatividad, confianza y aprendizaje autodirigidos para ser mejores ciudadanos y personas.

En relación a esta formación deben adquirir las personas a lo largo de su carrera educativa, la organización Mundial para la Salud, ha desarrollado una serie de destrezas que le permiten conducirse en la vida de forma hábil y competente, nombrando habilidades para la vida que están caracterizadas por ser competencias que desarrollan capacidades para vivir una vida sana y feliz. Las mismas promueven factores de protección frente a problemas psicosociales derivados del entorno; y ayudan a saber cómo enfrentarse a las exigencias y desafíos que la vida pone delante.

Al respecto la Organización Mundial de la Salud, expresa diez habilidades: 1. Autoconocimiento, 2. Manejo de emociones y sentimientos, 3. Manejo de la tensión y el estrés, 4. Comunicación asertiva, 5. Empatía, 6. Relaciones interpersonales, 7. Manejo de conflictos, 8. Toma de decisiones, 9. Pensamiento creativo, 10. Pensamiento crítico.

Sin duda alguna las competencias permiten al individuo aprender a conocer, aprender a hacer y a convivir, llevándolo a descubrir, despertar e incrementar su posibilidad de poder enfrentarse a situaciones concretas mediante la aplicación creativa, flexible y responsable de conocimientos habilidades y actitudes, y su relación con las habilidades para la 
vida es que éstas a su vez le permitirá enfrentarse de forma exitosa a las exigencias y desafíos de la vida diaria.

Los estudiantes en la etapa de educación universitaria adquieren nuevos conocimientos, inician con un proceso en el que han de estar seguros de su personalidad e identidad, ya que en esta fase existe gran influencia externa por parte de compañeros, docentes 0 personas con quienes socializan a diario. Ante este proceso, se requiere que los estudiantes se hayan enfrentado cada uno de los vectores propuestos por Chickering, con el fin de que cada etapa les brinde herramientas necesarias para abarcar las necesidades y exigencias propias universitarias las cuales requieren madurez, compromiso y propósitos definidos.

Durante la etapa universitaria se inicia con la búsqueda, internalización y transformación del entorno, permitiendo apertura a nuevas experiencias cognitivas y conductuales. Los estudiantes inician un proceso en donde se ve involucrada la comunidad estudiantil y la relación con sus pares caracterizando esta etapa como propia de un período en donde los jóvenes inician un proceso de socialización mucho más amplio, en el que se da una apertura a la comunicación no solo con jóvenes de sus carreras, de su edad o de su estatus social sino que se amplía este proceso a toda la comunidad estudiantil (Bittar, 2008)

Este aspecto no escapa de la realidad de los estudiantes del Instituto Universitario de Tecnología READIC UNIR, que al momento de culminar con sus estudios académicos se evidencian deficiencias en estas competencias que se gestan y amplían a partir de las unidades curriculares ética y desarrollo personal. Estas se imparten en el primer y quinto semestre y promueven en los estudiantes autoestima, manejo de emociones, consolidación de metas, entre otros aspectos, sin embargo cuando el estudiante se enfrenta con una realidad laboral a través de pasantías y prácticas se reflejan muestras mínimas de estas destrezas, las cuales de cierta forma van a dificultar la inclusión de los mismos en los procesos laborales.

Se planteó así como objetivo general analizar las competencias psicosociales de los estudiantes del Instituto Universitario de Tecnología READIC UNIR. Seguido de objetivos específicos como: Identificar el desarrollo de competencias intelectuales, sociales e interpersonales de los estudiantes universitarios; reconocer la competencia gestión de las emociones de los estudiantes universitarios; describir la competencia desarrollo de la autonomía y la interdependencia de los estudiantes universitarios; identificar la competencia desarrollo de relaciones interpersonales de los estudiantes universitarios; identificar la competencia establecimiento de la identidad de los estudiantes universitarios; reconocer la competencia desarrollo del propósito de los estudiantes universitarios, y describir la competencia desarrollo de la integridad de los estudiantes universitarios.

El estudio estuvo enmarcado en la línea de investigación orientación educativa, fundamentada en el contenido teórico con respecto a la concepción de competencias psicosociales de acuerdo a Chickering y Reisser (1993). Esta 
investigación se realizó durante las fecha octubre 2017 y marzo 2018.

La investigación se justificó porque posibilitó determinar a partir de fuentes fidedignas el nivel de desarrollo de las competencias que le permitirán incorporarse en un mundo laboral y la sociedad como trabajadores integrales, además de permanecer en el campo laboral a estudiantes universitarios; ofrece su contribución a partir de varios aspectos: desde el punto de vista teórico, aborda un tema por demás innovador, oportuno y pertinente, ya que en la actualidad se vislumbra la necesidad de desarrollo integral de los estudiantes y la preparación para el campo laboral, por ende en la investigación se recolectan las referencias teóricas que puedan medir los siete vectores que desarrollan las competencias psicosociales de los estudiantes universitarios, realizando una recopilación de diversas teorías.

Desde el aspecto metodológico, evidenciará la importancia de la elección del paradigma, el enfoque y método más adecuado para la investigación que se relaciona con hechos humanos $y / 0$ sociales, pero el aporte metodológico más importante es el diseño de un instrumento que es capaz de medir las competencias psicosociales de los estudiantes universitarios en cualquiera de los contextos, a su vez desde el punto de vista práctico, representa una posibilidad para conocer una realidad desde este enfoque; desde el desarrollo de las competencias de los estudiantes de Instituto Universitario de Tecnología Readic UNIR, ya que generara aportes a la institución para poder confrontar y emplear acciones que le permitan egresar estudiantes que cumplan con un desarrollo integral.

En cuanto al aporte social la presente investigación brinda una herramienta para el contexto en el cual opera la institución educativa objeto de estudio, con el propósito de que los estudiantes egresados al ingresar al campo laboral se desenvuelvan de forma óptima en su trabajo y sean un punto de transformación de la sociedad, sirviendo también de modelo a otros profesionales, los cuales permiten reconocer el nivel de desarrollo de las competencias psicosociales de los estudiantes que son objeto de estudio.

En el marco de contextualizar los aspectos fundamentales de la problemática de investigación se consideró oportuno cómo el Instituto Universitario de Tecnología Readic-UNIR presenta un modelo educativo basado en un sentido humano y orientado a servir, incorporando el espíritu de servicio a la formación ética y ciudadana de sus estudiantes. El gran compromiso se orienta a la formación integral de hombres y mujeres capaces de generar soluciones a los problemas que encara el siglo XXI en lo referido a la educación a través de un modelo por competencias desarrollando las habilidades y destrezas del ser, este diseño curricular va a dar respuesta a las necesidades de su colectividad enmarcándolo en el modelo progresista a través de experiencia educativa teórica y práctica formando personas que se conviertan en agentes de cambio dispuestos a ser a un más competitivos y responsable de su propia vida, de su realidad económica, política, y ecológica 
tanto en el plano personal con en el social y profesional.

Cabe destacar que la educación superior busca estar siempre a la vanguardia y en continuo aprendizaje actualizado con el propósito de formar ciudadanos no sólo desde el punto de vista técnico sino de una forma integral, que pueda sentir un mayor compromiso consigo mismo y su desarrollo personal, siendo personas libres, críticas, solidarias, capaces de incluirse en un mundo globalizado y sobre todo capaces de desarrollar habilidades que le permita aplicar los conocimientos adquiridos durante la carrera y que de esta manera pueda contar con la disposición de trabajar inmerso en una sociedad productiva y a la vez exigente para lograr constituirse en agentes de cambios sociales y positivos. Por ello se introduce la educación por competencias para responder esencialmente a la necesidad de alinear el mundo educativo con el mundo del trabajo.

Académicamente las competencias se entienden como actuaciones integrales para identificar, interpretar, argumentar y resolver problemas del contexto con idoneidad y ética, integrando el saber ser, el saber hacer y el saber conocer (Tobón, 2013). Por ende se puede plantear que las competencias son un conjunto articulado y dinámico de conocimientos, habilidades, actitudes y valores que toman parte activa en el desempeño responsable y eficaz de las actividades cotidianas dentro de un contexto determinado.

Para el estudio de las competencias psicosociales es preciso revisar la teoría de los siete vectores de Arthur Chickering y Reisser (1993) basada en las ideas de Erick Erickson (1959-1980). Esta teoría tiene como base el establecimiento de la identidad como aspecto fundamental para el desarrollo de los estudiantes durante sus años de preparación y formación en la universidad, aproximadamente entre los 18 y los 24 años de edad y ha servido de apoyo para investigaciones sobre la prosecución académica. Esta teoría fue validada por Soares y Almeida (2004).

Para esta investigación se toma la definición de este autor el cual plantea que las competencias psicosociales son un conjunto de habilidades, capacidades necesarias y susceptibles a ser desarrolladas que garantizan el éxito académico profesional del estudiante universitario.

Esta teoría propone el desarrollo de siete vectores como mapas para ayudar a determinar dónde están los estudiantes, cuáles caminos están tomando. El movimiento a lo largo de los vectores no requieren un secuencia lineal, esto significa que el avance de los estudiantes a través de los vectores puede variar el orden ya que el desarrollo de los estudiantes ocurre a ritmos diferentes, de tal forma que éste depende de las necesidades y circunstancias propias de cada uno. Avanzar a través de ellos otorga más conciencia, estabilidad, destreza, confianza e integración, ya que cada vector comprende fortalezas y debilidades para el crecimiento y para adaptarse cuando aparecen los problemas. A continuación se explican brevemente: 


\section{1.- Desarrollo de competencias} académicas individuales (intelectuales, sociales e interpersonales) cuyo sentido se manifiesta, de manera concreta, en la confianza con que los estudiantes asumen los retos que se les presentan y en alcanzar objetivos con éxito. Estos comprenden los siguientes:

a) Las competencias intelectuales es la habilidad que tiene el estudiante de usar la mente para ganar sofisticación intelectual, y desarrollar habilidades que ayuden en la comprensión y el análisis. No se refiere solo a memorizar sino en el desarrollo de las operaciones formales del pensamiento, para superar las debilidades académicas con las que ingresan los estudiantes a las universidades. Por ende estas se basan en habilidades menteestudiante siendo el contenido principal para su desarrollo.

b) Competencias sociales, tal como lo plantea Chickering (1993), se caracterizan por las habilidades de los estudiantes en el trabajo en grupo y la configuración de procesos de liderazgo, capacidad para tomar decisiones, ser directo con lo que quiere, sensible y empático en la comunicación con los otros.

c) Competencias interpersonales: se caracterizan por las habilidades de los estudiantes en el trabajo en grupo $y$ en equipos de estudios. Esta competencia implica: capacidad de escuchar, de cooperar y comunicarse, así como "trabajar sin problemas con un grupo, expresarse con asertividad, manifestando lo que necesita.

2.- Gestión de las emociones, concerniente al desarrollo de la capacidad para reconocer y aceptar las emociones, controlarlas y expresarlas adecuadamente; es decir, conciencia emocional y control emocional

3. Desarrollo de la autonomía, que apunta al reconocimiento y aceptación de la importancia de la interdependencia, incluyendo el desarrollo de la independencia emocional e instrumental. Al existir independencia emocional aumenta la autonomía por lo que la persona es capaz de desenvolverse autosuficientemente, abriendo paso a que el nivel de exigencias aumente estableciendo parámetros en los cuales se va a mostrar flexible.

4. Desarrollo de relaciones interpersonales, relacionada con la aceptación y la tolerancia de las diferencias individuales, incluyendo el establecimiento de relaciones íntimas; en este proceso los estudiantes deben establecer relaciones maduras, y con tolerancia, que sirva también de apoyo para la consecución de objetivos personales y profesionales.

5. Establecimiento de la identidad, se refiere a la autoaceptación en un sentido integral: apariencia, género y orientación sexual, continuidad de la identidad en diferentes situaciones e integración; en este vector se aspira que logre su definición personal, claridad de valores, aspecto fundamental para enfrentarse 
exitosamente a los cambios propio de la formación profesional.

\section{Desarrollo del propósito, importante} para la toma de decisiones profesionales, afirmación de intereses personales y el establecimiento de compromisos a largo plazo; en este vector, plantean que no se refiere a la culminación de la carrera sino más bien en el proceso de reafirmación vocacional que, por supuesto, es un elemento fundamental para la prosecución académica, es decir madurez vocacional.

\section{Desarrollo de la integridad,} “...entendida como la clarificación, compromiso y congruencia de los estudiantes con un conjunto de valores" (Blanco, 2005, p. 19). La integridad está relacionada por ende con los valores cívicos, familiares y creencias, demostradas en sus actitudes diarias permitiéndose desarrollar un ambiente apto, estableciendo un desarrollo integral.

\section{MÉTODO}

Desde el punto de vista metodológico para llevar a cabo este estudio se tomó el enfoque cuantitativo, puesto se utiliza primero para descubrir y refinar preguntas de investigación. A veces, pero no necesariamente, se prueban hipótesis (Hernández et al., 2010).

La investigación fue de tipo descriptiva, ya que el propósito de la misma buscó recopilar información de hechos o características reales en la organización. En síntesis, debido al enfoque y tipo de investigación, se seleccionó una serie de cuestiones, tal es el caso de la variable de competencias psicosociales de estudiantes universitarios del Instituto Universitario de Tecnología Readic UNIR; a fin de lograr describir esta variable en la muestra representativa.

Puesto que el desarrollo de este trabajo determinó las competencias psicosociales de estudiantes universitarios del Instituto Universitario de Tecnología Readic UNIR, el instrumento de la recolección de datos tuvo su asiento en el contacto directo del investigador con los sujetos involucrados en su ambiente.

Al respecto la población objeto de estudio se caracterizó por estudiantes del quinto semestre del Instituto Universitario de Tecnología Readic UNIR, en las cuales se indagaron sobre las competencias psicosociales adquiridas por estos estudiantes durante sus estudios universitarios.

Se empleó un muestreo no probabilístico de tipo intencional también llamado sesgado caracterizado por el hecho de obtener muestras representativas mediante la inclusión en la muestra de grupos típicos, por parte del investigador seleccionando directa e intencionadamente de los estudiantes, la cual estuvo conformada por un número significativo de estudiantes de cada una de las carreras.

Cabe destacar que para el cuestionario que estudió la variable competencias psicosociales se estructuró en una parte con ítems. Se empleó la escala de medición tipo Likert, que consiste en un conjunto de ítems presentados en forma de afirmaciones o juicios, ante los cuales se pide la reacción de los participantes, formulando proposiciones relativas a una serie de 
atributos de un objeto, a través del cual el entrevistado expresa su grado tanto de acuerdo como de desacuerdo en una escala de varias categorías, con cinco alternativas de respuestas: Siempre, Casi Siempre, Algunas veces, Casi nunca y Nunca, con una ponderación del 5 al 1.

En este caso, la validez del instrumento se sometió a un juicio de expertos, con la finalidad de determinar si presentan los contenidos y criterios suficientes para considerarlos válidos expresando sus opiniones y sugerencias sobre los ítems formulados, determinando la organización, claridad, redacción, técnicas, pertinencia y coherencia entre las variables, ítems y objetivos. A tales fines el cuestionario fue sometido al juicio de cinco expertos quienes emitieron su evaluación pertinente del mismo.

Se aplicó una prueba piloto para determinar la validez interna del mismo; la cual se llevó a cabo a partir del cálculo de la confiabilidad. En este sentido, los resultados de las pruebas estadísticas para establecer el índice de confiabilidad determinó para el cuestionario un coeficiente Alfa Crombach de 0,892; resultando ser Muy alta.

Para la investigación, el plan de análisis de datos incluyó el uso de la estadística descriptiva a partir de la distribución de frecuencias absolutas (N) $y$ relativas o porcentuales (\%) para conocer la tendencia en las respuestas de los sujetos que conforman el contexto de estudio.

\section{RESULTADOS}

De la dimensión desarrollo de competencias resultó que el $80 \%$ de los encuestados, poseen en un rango alto estas competencias, por su parte el $20 \%$ medianamente poseen esta dimensión. La media de la dimensión es de 4.10 considerándose su competencia en un nivel "Alto", lo que revela que los estudiantes presentan competencias intelectuales, sociales e interpersonales, asumiendo los retos a los que se enfrentan. (Ver Tabla 1)

Tabla 1. Baremo para interpretar los indicadores: Dimensiones y Variable en estudio

$\begin{array}{cc}\text { CATEGORÍAS } & \text { PUNTUACION } \\ 1,00-2,33 & \text { Baja } \\ 2,34-3,66 & \text { Media } \\ 3,67-5,00 & \text { Alta }\end{array}$

Fuente: Elaboración propia.

Chickering y Reisser (1993) expresan que el desarrollo de competencias académicas individuales (intelectuales, sociales e interpersonales), se manifiesta, de manera concreta, en la confianza con que los estudiantes asumen los retos que se les presentan y en alcanzar objetivos con éxito. Por ende se evidenció que los estudiantes encuestados presentan seguridad al asumir retos académicos en su vida y con confianza de asumir los nuevos objetivos profesionales a los que se enfrentan. 
Los resultados de la dimensión gestión de las emociones arrojaron que el $92 \%$ de los encuestados, poseen en un rango alto competencias para gerenciar sus emociones, por su parte el $8 \%$ medianamente poseen esta dimensión. La media de la dimensión es de 4.20 considerándose su competencia en un nivel "Alto", lo que revela que los estudiantes presentan competencias para reconocer y aceptar las emociones, controlarlas y expresarlas adecuadamente.

La gestión de las emociones (valorando la propuesta de Yancovic, 2011), concerniente al desarrollo de la capacidad para reconocer y aceptar las emociones, controlarlas y expresarlas adecuadamente; es decir, conciencia emocional y control emocional, según Chickering y Reisser (1993 c. p. Forney, Patton y Renn, 2010).

Para la dimensión autonomía e independencia, se obtuvo que el $96 \%$ de los encuestados posee en un rango alto autonomía e independencia mostrándose autosuficiente, por su parte el $4 \%$.

La media de la dimensión es de 4.52 considerándose su competencia en un nivel "Alto", lo que revela que los estudiantes poseen la capacidad para ser autosuficientes, dejar un lugar y tener éxito en otra, logran ser críticos y decididos en los retos que enfrentan.

Chickering y Reisser (1993) expresan que el desarrollo de la autonomía, que apunta al reconocimiento y aceptación de la importancia de la interdependencia, incluyendo el desarrollo de la independencia emocional e instrumental. Al existir independencia emocional aumenta la autonomía por lo que la persona es capaz de desenvolverse autosuficientemente, abriendo paso a que el nivel de exigencias aumente estableciendo parámetros en los cuales se va a mostrar flexible. Por ende se evidencia que los jóvenes poseen gran autonomía e independencia al momento de desarrollarse como estudiantes universitarios.

Los resultados de la dimensión relaciones interpersonales fueron que el $72 \%$ de los encuestados posee en un rango alto competencias para mantener relaciones interpersonales acertadas, por su parte el $28 \%$ medianamente poseen esta dimensión. La media de la dimensión es de 4.02 considerándose su competencia en un nivel "Alto", lo que revela que los estudiantes presentan aceptación, tolerancia de las diferencias individuales, incluyendo el establecimiento de relaciones íntimas.

Chickering y Reisser (1993) expresan que el desarrollo de relaciones interpersonales están vinculadas con la aceptación y la tolerancia de las diferencias individuales, incluyendo el establecimiento de relaciones íntimas; en este proceso los estudiantes deben establecer relaciones maduras y con tolerancia, que sirvan también de apoyo para la consecución de objetivos personales y profesionales. Al encontrar emprender la etapa universitaria los estudiantes establecen relaciones interpersonales con personas que presentan diferentes creencias lo que genera mayor tolerancia a la diferencia, por ende deben mostrarse con una comunicación basada en la asertividad, y ante esto se evidencia como estos jóvenes 
escogerán personas con las que generaran vínculos íntimos de amistad e incluso de romance.

Los resultados de la dimensión establecimiento de la identidad expresaron que el $88 \%$ de los encuestados, poseen en un rango alto Autoaceptación y autoconcepto, por su parte el $12 \%$ medianamente poseen esta dimensión. La media de la dimensión es de 4.57 considerándose su competencia en un nivel "Alto", lo que revela que los estudiantes poseen Autoaceptación en un sentido integral: apariencia, género y orientación sexual, además de continuidad de la identidad en diferentes situaciones e integración.

El establecimiento de la identidad se aspira que logre su definición personal, claridad de valores, aspecto fundamental para enfrentarse exitosamente a los cambios propios de la formación profesional. La identidad puede ser considerada como un determinante ante la conducta de las personas, ya que gracias a su desarrollo la persona va a proyectar su comportamiento, dependiendo de sus valores y costumbres (Chickering y Reisser, 1993). Por ende los jóvenes han alcanzado en un rango alto una identidad personal definida donde están claros en sus creencias, costumbres, orientación sexual, gustos y personalidad.

Los resultados de la dimensión desarrollo de propósito fueron que el 96\% de los encuestados tienen en un rango alto la competencia para el desarrollo de propósito, por su parte el $4 \%$ medianamente poseen esta dimensión. La media de la dimensión es de 4.53 considerándose su competencia en un nivel "Alto", lo que revela que los estudiantes toman de decisiones profesionales, afirman sus intereses personales y establecen compromisos a largo plazo.

El desarrollo del propósito es importante para la toma de decisiones profesionales, afirmación de intereses personales y el establecimiento de compromisos a largo plazo. En este vector, plantean que no se refiere a la culminación de la carrera sino más bien en el proceso de reafirmación vocacional que, por supuesto, es un elemento fundamental para la prosecución académica, es decir madurez vocacional (Chickering y Reisser, 1993). Los estudiantes han desarrollado este vector estableciéndose metas a futuro por las cuales velarán para su cumplimiento, se muestran curiosos ante el mundo del trabajo, convirtiéndose en la fase primaria del desarrollo individual.

Los resultados de la dimensión desarrollo de la integridad fueron que el $96 \%$ de los encuestados, poseen en un rango alto la competencia para el desarrollo de la integridad, por su parte el $4 \%$ medianamente poseen esta dimensión. La media de la dimensión es de 4.62 considerándose su competencia en un nivel "Alto", lo que revela que los estudiantes desarrollan su propio conjunto de valores e intereses.

El Desarrollo de la integridad, “...entendida como la clarificación, compromiso y congruencia de los estudiantes con un conjunto de valores" (Blanco, 2005, p. 19), está relacionada por ende con los valores cívicos, familiares y creencias, demostradas en 
sus actitudes diarias permitiéndose desarrollar un ambiente apto, estableciendo un desarrollo integral, aspecto que son altamente demostradas por los estudiantes universitarios desarrollando su propio conjunto de valores e intereses por lo que tienden a alejarse de las creencias dogmáticas con las que crecieron y empiezan a desarrollar las suyas propias.

La variable competencias psicosociales obtuvo los siguientes resultados el $92 \%$ de los encuestados, es decir 23 poseen en un rango alto la competencias psicosociales, por su parte el $8 \%$ que equivale a 2 estudiantes medianamente poseen esta dimensión. La media de la dimensión es de 4.36 considerándose su competencia en un nivel "Alto", lo que revela que los estudiantes poseen un grado de suficiencia y responsabilidad para avanzar por la universidad y realizar ciertas tareas psicológicas y sociales.

Se entiende por competencia psicosocial lo que el estudiante es capaz de ejecutar, es el grado de suficiencia y responsabilidad para avanzar por la universidad y realizar ciertas tareas psicológicas y sociales que facilitan el desarrollo la responsabilidad social y el aprendizaje autodirigido a través de destrezas tales como: competencias interpersonales, manejo de emociones y el desarrollo del propósito en la vida. (Chickering y Reisser, 1993)

Al respecto los estudiantes se muestran en muchas formas con competencias psicosociales que otorgan más conciencia, estabilidad, destreza, confianza e integración, ya que cada vector comprende fortalezas y debilidades para el crecimiento y para adaptarse cuando aparecen los problemas.

\section{CONCLUSIONES}

Analizados y discutidos los resultados obtenidos en esta investigación sobre competencias psicosociales en estudiantes universitarios, se presentan a continuación las palabras de cierre, de conformidad a los objetivos inicialmente propuestos.

En el objetivo 1 que declara identificar el desarrollo de competencias intelectuales, sociales e interpersonales de los estudiantes universitarios, se concluye que los estudiantes desarrollan las competencias intelectuales, sociales e interpersonales de forma que asumen los retos a los que se enfrentan, alcanzando con éxito los objetivos académicos que se plantean.

Con respecto al objetivo 2 que cita reconocer la competencia gestión de las emociones de los estudiantes universitarios, se afina que los estudiantes presentan competencias para reconocer y aceptar las emociones, controlarlas y expresarlas adecuadamente, además demostrándose empáticos al momento de socializar con las demás personas.

El objetivo 3 que declara describir la competencia desarrollo de la autonomía y la interdependencia de los estudiantes universitarios, reveló que los estudiantes poseen la capacidad para ser autosuficientes, dejar un lugar y tener éxito en otra, logran ser críticos y decididos en los retos que enfrentan, mostrándose flexible ante los cambios con independencia tanto instrumental como emocional. 
Los resultados del objetivo 4 Identificar la competencia desarrollo de relaciones interpersonales de los estudiantes universitarios, develaron la siguiente conclusión: los estudiantes presentan aceptación, tolerancia de las diferencias individuales, incluyendo el establecimiento de relaciones íntimas, desarrollando relaciones interpersonales maduras, que promueven una comunicación asertiva y tolerancia a las diferencias.

Los resultados del objetivo 5 que cita identificar la competencia establecimiento de la identidad de los estudiantes universitarios, dejaron ver que los estudiantes poseen autoaceptación en un sentido integral: apariencia, género $y$ orientación sexual, además de continuidad de la identidad en diferentes situaciones e integración, sintiéndose claros con sus están claros en sus creencias, costumbres, gustos y personalidad.

Con respecto al objetivo 6 que declara reconocer la competencia desarrollo del propósito de los estudiantes universitarios se concluye que los estudiantes han desarrollado este vector estableciéndose metas a futuro, se muestran curiosos ante el mundo del trabajo, convirtiéndose en la fase primaria del desarrollo individual, es decir toman de decisiones profesionales, afirman sus intereses personales $y$ establecen compromisos a largo plazo.

En base a los resultados del objetivo 7 describir la competencia desarrollo de la integridad de los estudiantes universitarios, se afina que el comportamiento de los estudiantes es congruente con los valores y creencias que poseen y los requeridos para el ejercicio de la profesión que estudia, demostrados en sus comportamientos personales y en sus intereses.

Por último, se concluye que en cuanto al objetivo general: analizar las competencias psicosociales de los estudiantes universitarios del Instituto Universitario de Tecnología READIC UNIR, los estudiantes se muestran en muchas formas con competencias psicosociales que otorgan más conciencia, estabilidad, destreza, confianza e integración, ya que cada vector comprende fortalezas y debilidades para el crecimiento y para adaptarse cuando aparecen los problemas, cumpliendo con las tareas psicológicas y sociales que acogen la vida universitaria.

\section{REFERENCIAS}

Bittar, M. (2008). Investigación sobre burnout y estilos de personalidad en estudiantes universitarios. Recuperado de

http://fci.uib.es/digitalAssets/177/177 915_2.pdf

Chickering A., y Reisser, L. (1993). Education and identity (2a Ed.). San Francisco: Jossey-Bass Publishers. pp. 45- 237

Forney, D. S., Guido, F. M., Patton, L. D., y Renn, K. A. (2010). Racial identity development. Student development in college: Theory, research and practice, 260-261

Hernández, Fernández y Baptista (2010). Metodología de la Investigación. México: McGraw-Hill

Tobón, S. (2013). Formación Basada en Competencias. Colombia. Ediciones ECOE

UNESCO (2009). Conferencia Mundial sobre la Educación Superior - 2009: La nueva dinámica de la educación superior y la investigación para el 
cambio social y el desarrollo. Paris, Francia. Recuperado de: http://www.unesco.org/education/WCH E2009/comunicado es.pdf

Yankovic B. El desarrollo social y afectivo en los niños de primer ciclo básico.
Seminario de Tesis Facultad de Educación [Internet]. Santiago: Universidad Mayor 